\title{
ADICIONES A LA FLORA MARINA DEL CARIBE MEXICANO
}

\author{
Luis E. Aguilar Rosas \\ Universidad Autónoma de Baja California \\ Instituto de Investigaciones Oceanologicas \\ Apdo. Postal 453, Ensenada, Baja California, México \\ Mafco A. Aguilar Rosas \\ Antonio Gomez Pedroso Cedillo \\ Jesus Antonio Fernandez Prieto \\ Centro de Investigaciones de Quintana Roo, A. C. \\ A. P. 424, Chetumal, Quintana Roo, México
}

\section{RESUMEN}

Se determinan 21 especies y una variedad de algas marinas bentónicas como nuevos registros para el Caribe Mexicano en las costas de Quintana Roo, México.

\section{ABSTRACT}

21 species and one variety of marine benthic algae are reported for the first time for the Mexican Caribbean on the coast of Quintana Roo, México.

\section{INTRODUCCION}

El Caribe mexicano, en las costas de Quintana Roo, ha sido objeto de estudios ficológicos los cuales prácticamente se inician con el trabajo de Huerta-Múzquiz (1958) en Cozumel e Isla Mujeres. Taylor (1972) cita algunas especies de algas colectadas durante la expedición Smithsonian-Bredin a las costas de la Península de Yucatán. GarzaBarrientos (1975) realiza un estudio sobre la flora marina en varias localidades a lo largo de la costa de Quintana Roo. Huerta-Múzquiz y Garza-Barrientos (1980) presentan una lista de 200 especies de algas, incluyendo cianoficeas, en su trabajo de las algas de la región de Chetumal, Xcalak y Banco Chinchorro, en el sur del estado.

En la compilación de información de la flora marina de la Península de Yucatán (Huerta-Múzquiz et al., 1987) encuentran un total de 412 especies y taxa infraespecíficos de algas para la costa de Quintana Roo, incluyendo las islas Contoy, Mujeres y Cozumel asi como Banco Chinchorro; el material ficológico correspondiente se encuentra depositado en el herbario ENCB.

Colectas recientes llevadas a cabo en los alrededores de Puerto Morelos y en la Bahía de la Ascensión indican la presencia de 21 especies y una variedad de algas marinas que representan nuevos registros para el Caribe Mexicano en las costas de Quintana Roo. 


\section{MATERIALES Y METODOS}

Se realizaron colectas de algas en los alrededores de Puerto Morelos (Fig. 1) y en la Bahía de la Ascensión (Fig. 2), en la zona infralitoral utilizando buceo SCUBA.

Con el fin de abarcar el mayor número de ambientes posibles, se localizaron 10 sitios de muestreo a lo largo de aproximadamente $18 \mathrm{~km}$ de costa en Puerto Morelos y en Bahía de la Ascención otros 12 distribuidos a lo largo de la bahía.

Las especies fueron separadas en el laboratorio e identificadas siguiendo principalmente los trabajos de Börgesen (1913-1920), Taylor (1960), Chapman (1961, 1963), Joly (1965, 1967), Oliveira-Filho (1969) y Cordeiro-Marinho (1978).

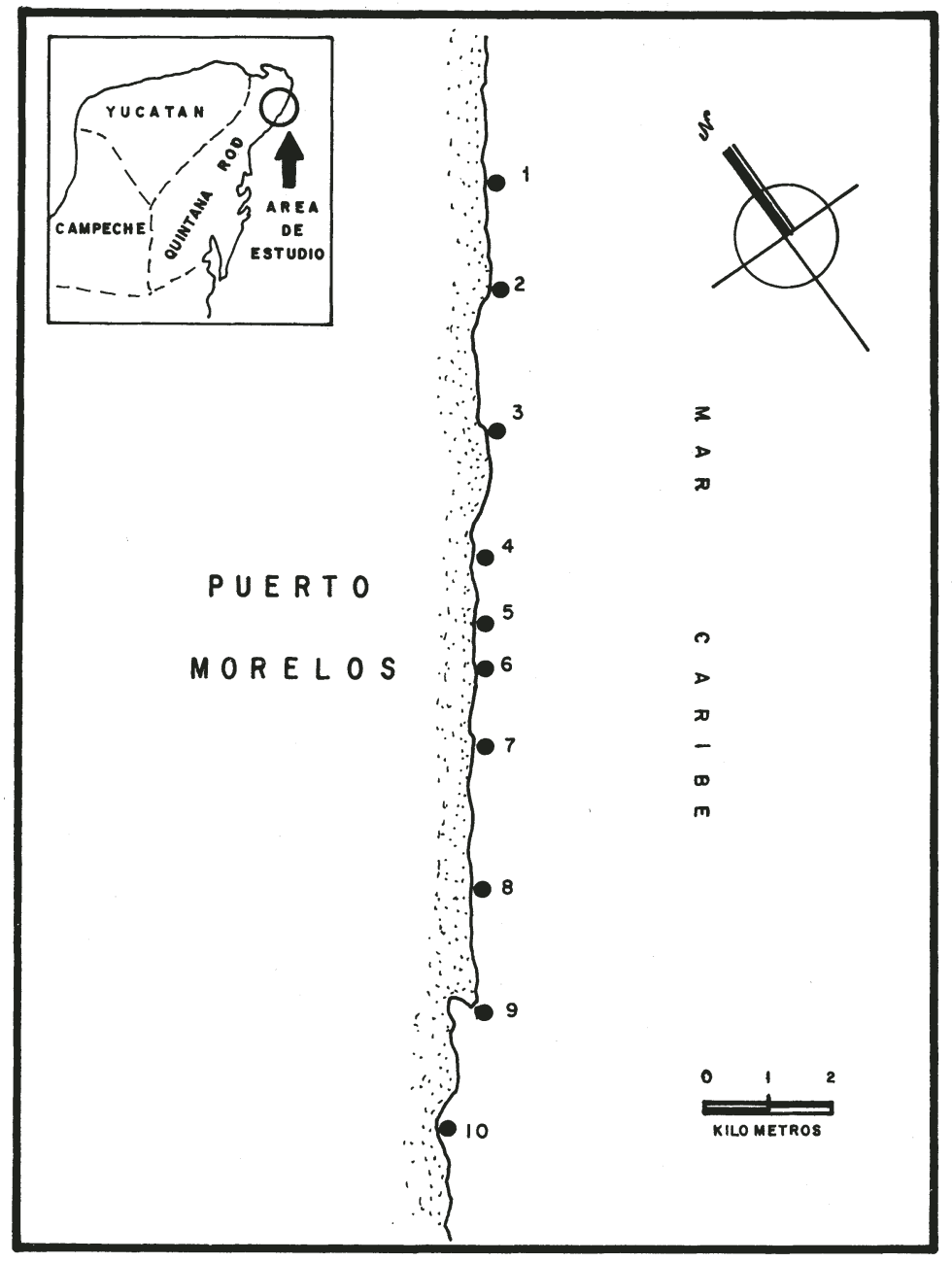

Fig. 1. Localización del área de estudio y sitios de muestreo en Puerto Morelos, Quintana Roo. 


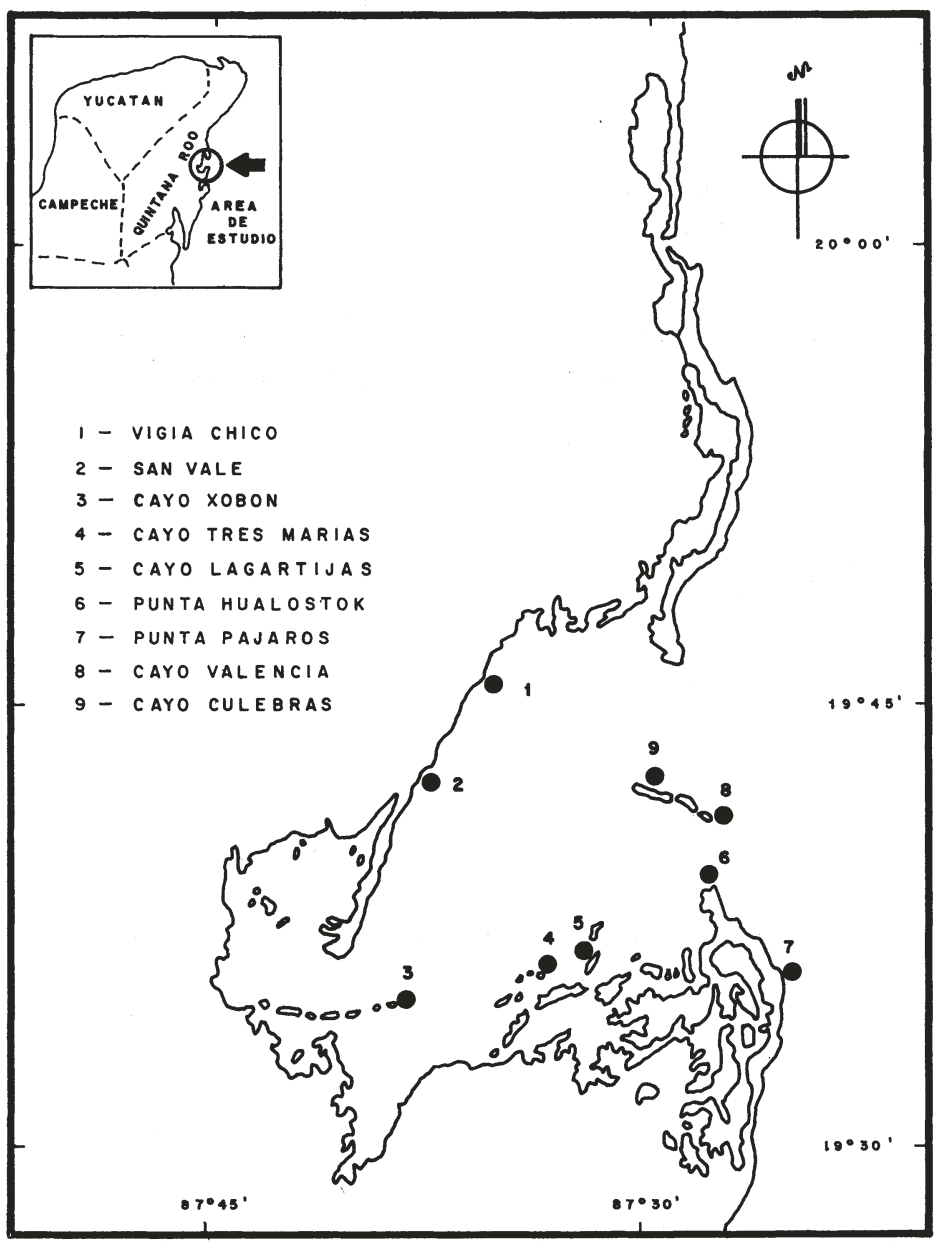

Fig. 2. Localización del área de estudio y sitios de muestreo en la Bahía de la Ascención, Quintana Roo.

\section{RESULTADOS}

La siguiente lista sistemática fue preparada siguiendo el esquema utilizado por Wynne (1986) e incluye localidad, número y fecha de colecta. Las colectas fueron realizadas por Antonio Gómez-Pedroso (AGP) y Luis E. Aguilar-Rosas, en Puerto Morelos, y por Jesús Fernández Prieto (JFP) y Luis E. Aguilar-Rosas en Bahía de la Ascensión.

De las 21 especies encontradas dos ya hablan sido registradas para la costa Atlántica de México: Siphonocladus rigidus para Arrecife Alacranes, Yucatán, e Hypneocolax stellaris para Campeche por Huerta-Múzquiz et al. (1987). 


\section{CHLOROPHYTA}

Siphonocladales

Siphonocladaceae

Siphonocladus tropicus (P. \& H. Crouan) J. Agardh

Material revisado: Cayo Xobon, JFP-417, 25.VI.86.

Siphonocladus rigidus Howe

Material revisado: Puerto Morelos-4, AGP-763, 10.IV.86; Puerto Morelos-2, AGP-1410, 22.VI.86; Vigía Chico, JFP-208, 30.IV.86; Vigla Chico, JFP-299, 24.VI.86.

Cladophorales

Cladophoraceae

Bryopsis halliae W. Taylor

Material revisado: Cayo Valencia, JFP-175, 30.IV.86.

Cladophora jongiorum Van den Hoek

Material revisado: Cayo Lagartijas, JFP-248, 31.IV.86.

Caulerpales

Caulerpaceae

Caulerpa lanuginosa J. Agardh

Material revisado: Puerto Morelos-6, AGP-470, 23.II.86; Puerto Morelos-2, AGP-899, 11.IV.86; Puerto Morelos-3, AGP-921, 11.IV.86; Puerto Morelos-6, AGP-659, 10.IV.86; Puerto Morelos-6, AGP-1050, 18.VI.86; Puerto Morelos-1, AGP-1227, 20.VI.86; Puerto Morelos-5, AGP-1352, 21.VI.86.

\section{Udoteaceae}

Halimeda lacrimosa Howe

Material revisado: Puerto Morelos-1, AGP-346, 21.II.86; Puerto Morelos-3, AGP-291, 20.1I.86; Puerto Morelos-4, AGP-256, 19.II.86; Puerto Morelos-5, AGP-428, 22.II.86; Puerto Morelos-7, AGP-236, 17.II.86; Puerto Morelos-1, AGP-831, 11.IV.86; Puerto Morelos-3, AGP-967, 16.IV.86; Puerto Morelos-4, AGP-722, 10.IV.86; Puerto Morelos-7, AGP-579, 9.IV.86; Puerto Morelos-1, AGP-1210, 20.VI.86; Puerto Morelos-4, AGP-1333, 20.VI.86;

Puerto Morelos-7, AGP-996, 18.VI.86; Puerto Morelos-10, AGP-1138, 19.VI.86. 


\section{PHAEOPHYTA}

Chordariales

Chordariaceae

Cladosiphon occidentalis Kylin

Material revisado: Puerto Morelos-7, AGP-212, 17.II.86.

\section{RHODOPHYTA}

Corallinales

Corallinaceae

Haliptilon subulatum (Ellis \& Solander) Johansen

Material revisado: Puerto Morelos-9, AGP-150, 17.II.86.

Gigartinales

Hypneaceae

Hypneocolax stellaris Börgesen

Material revisado: Cayo Valencia, JFP-501, 25.VII.86.

Nota: Esta especie ha sido citada para Campeche por Huerta-Múzquiz et al. (1987).

Rhodymeniales

Champiaceae

Gastroclonium parvum (Hollenberg) Chang \& Xia

Material revisado: Puerto Morelos-2, AGP-403, 22.II.86; Puerto Morelos-7, AGP-587, 9.IV.86; Puerto Morelos-4, AGP-1329, 20.VI.86; Cayo Lagartijas, JFP-250, 30.IV.86; Cayo Tres Marlas, JFP-548, 25.VII.86; Punta Hualostok, JFP-347, 26.II.86; Cayo Tres Marias, JFP-279, 24.VI.86; Punta Pájaros, JFP-449, 3.VII.86; Cayo Valencia, JFP-78, 27.II.86; Punta Hualostok, JFP-163, 30.IV.86.

Nota: Chang y Xia (1978), incluyen al género Coeloseira como sinónimo taxonómico de Gastroclonium.

Rhodymeniaceae

Botryocladia pyriformis (Börgesen) Kylin

Material revisado: Vigía Chico, JFP-327, 25.II.86.

Nota: Recientemente Mateo-Cid y Mendoza-González (1991), registran esta especie para la Isla Cozumel. 
Ceramiales

Ceramiaceae

Antithamnion cruciatum (C. Agardh) Nägeli

Material revisado: Puerto Morelos-4, AGP-1313, 20.VI.86.

Callithamnion cordatum Börgesen

Material revisado: Puerto Morelos-2, AGP-407, 22.II.86.

Grallatoria reptans Howe

Material revisado: Puerto Morelos-9, AGP-70, 17.II.86.

Wrangelia penicillata C. Agardh

Material revisado: Puerto Morelos-8, AGP-1182, 19.VI.86.

Nota: Recientemente Mateo-Cid y Mendoza-González (1991), registran esta especie para la Isla Cozumel.

Dasyaceae

Eupogodon antillarum (Howe) P.C. Silva

Material revisado: Puerto Morelos-1, AGP-785, 11.IV.86; Puerto Morelos-3, AGP-974, 16.IV.86; Puerto Morelos-9, AGP-549, 8.IV.86; Puerto Morelos-1, AGP-1249, 20.VI.86;

Puerto Morelos-5, AGP-1362, 21.VI.86; Puerto Morelos-9, AGP-1158, 19.VI.86.

Rhodomelaceae

Chondria platyramea Joly \& Ugadim

Material revisado: Puerto Morelos-10, AGP-22, 15.11.86; Puerto Morelos-7, AGP-1432, 17.II.86; Puerto Morelos-3, AGP-1268, 20.VI.86; Puerto Morelos-10, AGP-1121, 19.VI.86; Cayo Valencia, JFP-180, 30.IV.86; JFP-492, 25.VII.86.

Lophosiphonia cristata Falkenberg

Material revisado: Puerto Morelos-1, AGP-799, 11.IV.86; Puerto Morelos-3, AGP-951, 16.IV.86.

Polysiphonia breviarticulata (J. Agardh) Zanardini

Material revisado: Puerto Morelos-3, AGP-950, 16.IV.86; Cayo Valencia, JFP-77, 27.II.86; Cayo Culebras, JFP-101, 27.II.86; Cayo Lagartijas, JFP-246, 30.IV.86; Cayo Tres Marias; JFP-263, 30.IV.86; Cayo Valencia, 316, 25.II.86; Cayo Xobón, JFP-380, 26.II.86; Cayo Lagartijas, JFP-389, 26.II.86; Cayo Tres Marias, JFP-415, 26.II.86.

Polysiphonia eastwoodae Setchell \& Gardner

Material revisado: Puerto Morelos-9, AGP-122, 17.II.86; Puerto Morelos-1, AGP-882, 11.IV.86. 
Polysiphonia scopulorum var. villum (J. Agardh) Hollenberg

Material revisado: Puerto Morelos-3, AGP-324, 20.II.86; Puerto Morelos-2, AGP-910, 11.IV.86; Puerto Morelos-1, AGP-1229, 20.VI.86; Punta Hualostok, JFP-1335, 30.IV.86; San Vale, JFP-222, 30.IV.86; Vigia Chico, JFP-318, 25.II.86; Cayo Tres Marías, JFP-541, 25.VII.86.

\section{AGRADECIMIENTOS}

Los autores agradecen al Centro de Investigaciones de Quintana Roo, A. C. y al Consejo Nacional de Ciencia y Tecnología por el apoyo financiero para la realización del presente trabajo. Se dan las gracias asimismo al Instituto de Investigaciones Oceanologicas de la Universidad Autónoma de Baja Califonia, por el apoyo logístico, a la Bióloga Catalina Mendoza de la Escuela Nacional de Ciencias Biológicas del Instituto Politécnico Nacional, por sus comentarios, y al Sr. Ramón Moreno por la elaboración de las figuras.

\section{LITERATURA CITADA}

Börgesen, F. 1913. The marine algae of the Danish West Indies. Part 1. Chlorophyceae. Dansk. Bot. Arkiv $1(4): 1-158+2,126$ figs, 1 mapa.

Börgesen, F. 1914. The marine algae of the Danish West Indies. Part 2. Phaeophyceae. Dansk. Bot. Arkiv 2(2): $1-66+2,44$ figs.

Börgesen, F. 1915-1920. The marine algae of the Danish West Indies. Part 3. Rhodophyceae. Dansk. Bot. Arkiv 3(a-f): 1- 504, 435 figs.

Cordeiro-Marinho, M. 1978. Rodoficeas bentónicas marinhas do Estado de Santa Catarina. Rickia 7: 1243.

Chang, C. F., y B. M. Xia. 1978. A new species of Gastroclonium from the Xisha Island. Oceanologia Limnnologia Sinica 9: 213-214.

Chapman, V. J. 1961. The marine algae of Jamaica. Part 1: Myxophyceae and Chlorophyceae. Bull. Inst. Jamaica, Sci. Ser. 12(1): 1-159.

Chapman, V. J. 1963. The marine algae of Jamaica. Part 2: Phaeophyceae and Rhodophyceae. Bull. Inst. Jamaica, Sci. Ser. 12(2): 1-201.

Garza-Barrientos, M. A. 1975. Primeras consideraciones referentes sobre la flora marina del sureste de la República Mexicana. Memorias del II Simposio Latinoamericano sobre Oceanografía Biológica. Universidad de Oriente. Cumaná, Venezuela. Vol. I. 25 pp.

Huerta-Múzquiz, L. 1958. Contribución al conocimiento de las algas de los bajos de la Sonda de Campeche, Cozumel e Isla Mujeres. An. Esc. Nac. Cienc. Biol. Méx. 9(1-4): 115-123, 6-9 figs.

Huerta-Múzquiz, L. y M. Garza-Barrientos. 1980. Contribución al conocimiento de la flora marina de la parte sur del litoral de Quintana Roo, México. An. Esc. Nac. Cienc. Biol. Méx. 23: 25-44.

Huerta-Múzquiz, L., A. C. Mendoza-González y L. E. Mateo-Cid. 1987. Avance sobre un estudio de las algas marinas de la Península de Yucatán. Phytologia 62: 23-53.

Joly, A. B. 1965. Flora marinha do littoral Norte do Estado de Såo Paulo e regiones circunvizinhas. Boletin Fac. Fil. Cienc. e Letras da Universidade de Sáo Paulo. Botanica 21: 1-393.

Joly, A. B. 1967. Generos de algas marinhas da costa Atlantica Latinoamericana. Universidade de Sâo Paulo, Sâo Paulo. 464 pp.

Mateo-Cid, L. E. y A. C. Mendoza-González. 1991. Algas marinas bénticas de la Isla Cozumel, Quintana Roo, México. Acta Bot. Mex. 16: 57-87. 
Oliveira-Filho, E. C. 1969. Algas marinhas do Sul do Estado do Espiritu Santo (Brasil). I. Ceramiales. Boletin Fac. Fil. Cienc. e Letras da Universidade de Sáo Paulo. Botanica 26: 1-277.

Taylor, W. R. 1960. Marine algae of the eastern tropical and subtropical coast of the Americas. Univ. Mich. Press. Ann Arbor. 870 pp.

Taylor, W. R. 1972. Marine algae of the Smithsonian-Bredin expedition to Yucatan. 1960. Bull. Mar. Sci. 22(1): 34-44.

Wynne, M. J. 1986. A checklist of benthic marine algae of the tropical and subtropical western Attantic. Can. Journ. Bot. 64: 2239-2281. 